\title{
Caracterización térmica y mecánica de bloque de concreto
}

\section{Thermal and mechanical characterization of concrete block}

Mario F. Bustamante Crespo

Universidad Internacional SEK, Ecuador

Javier Martínez-Gómez

Universidad Internacional SEK, Ecuador

Instituto Nacional de Eficiencia Energética y Energías Renovables - INER, Ecuador

José Macías

Instituto Nacional de Eficiencia Energética y Energías Renovables - INER, Ecuador

Autor para correspondencia: javier.martinez@uisek.edu.ec

Fecha de recepción: 12 de abril de 2018 - Fecha de aceptación: 01 noviembre de 2018

Resumen: El diseño para construcciones de viviendas en Ecuador, en su mayoría se priorizan las resistencias mecánicas, dejando de lado el análisis de las prestaciones térmicas. Esto provoca un incremento en el consumo de energía, por lo cual se generan pérdidas monetarias y se produce un efecto negativo en el medioambiente. Una alternativa a esta situación es la promoción del uso de materiales y técnicas de construcción de mejor rendimiento energético y que a su vez puedan ofrecer un ambiente de confort. Para el presente estudio, se caracterizó tanto las propiedades mecánicas como las térmicas de muestras de concreto, fabricados en la región de Pichincha de ecuador. Se determinaron magnitudes como resistencia a la compresión, contenido de humedad y conductividad térmica. Los resultados muestran que los bloques del sector de Amagüaña poseen menor resistencia a compresión en base a la norma INEN. En los resultados de la conductividad térmica se mostraron valores menores a los de las referencias consultadas. Esto demuestra que tienen mejores características aislantes.

Palabras claves: bloque de concreto; material de construcción; conductividad térmica; propiedades mecánicas; Ecuador

Abstract: The design for housing construction in Ecuador, mostly prioritizes mechanical resistance. However, thermal considerations are overlooked. This causes an increase in energy consumption, which leads to monetary losses and a negative effect on the environment. A solution to these events is to promote the construction of self-sustaining housing, and for this it is necessary to use materials that are energy efficient that can offer a comfortable environment. In the present project the concrete mechanical and thermal properties of the Pichincha region of Ecuador were evaluated. Magnitudes such as compressive strength, moisture content and thermal conductivity were determined. The results show that the blocks of the Amagüa sector have a lower resistance to compression based on the INEN standard. In the results of the thermal conductivity are lower values than those of the consulted recommendations. This shows that they have better insulating characteristics.

Key words: concrete block; construction material; thermal conductivity; mechanical properties; Ecuador 


\section{Introducción}

El sector de la construcción fue identificado como uno de los sectores clave para lograr reducciones drásticas en la emisión de gases de efecto invernadero. Por un lado, los edificios son responsables del 40\% del consumo de energía y del 36\% de las emisiones de CO2 en la Unión Europea (UE) (Commissie, E., 2011). Mientras que los edificios nuevos en general necesitan menos de tres a cinco litros de combustible para calefacción por metro cuadrado por año, los edificios más viejos consumían alrededor de 25 litros en promedio (ECOFYS, 2014). Por otro lado, el Departamento de Energía de EE. UU. (DOE) está incentivando el desarrollo del rendimiento energético a través de la promoción de tecnologías, sistemas y prácticas eficientes, asequibles y de alto impacto. El objetivo a largo plazo de las tecnologías de construcción del DOE es reducir el uso de energía en un 50\%, en comparación con la línea base de 2010 (ASHRAE 90.1, 2007).

En el caso de Ecuador según el último Censo de Población y Vivienda, el Ecuador cuenta con una población de 14.306.876 millones de habitantes y 6’021.053 de viviendas (INER, 2013). De las viviendas registradas, el 94,75\% se encuentran ubicadas en la región costa y sierra del país.

Es preciso señalar que a nivel nacional en la fabricación de las viviendas se utilizan diferentes materiales, los mismos que varían en función de la región climática. Cabe indicar que a nivel nacional los materiales utilizados en la construcción de las viviendas se utilizan sin considerar criterios de confort térmico pues independientemente del nivel socioeconómico de la población, existe un desconocimiento sobre estos conceptos y en la mayoría de casos los materiales que se utilizan consideran únicamente el costo de inversión inicial. Actualmente, el país no cuenta con un laboratorio que permita realizar el estudio y análisis de propiedades térmicas de materiales y sistemas estructurales que respondan a una realidad local, tampoco tiene estudios que aporten en la generación de una base de datos de caracterización térmica de materiales, situación que ha generado dependencia tecnológica puesto que los datos existentes son referencias internacionales que no se ajustan a la realidad de los materiales utilizados en el país (INER, 2013).

A nivel nacional, la única información existente sobre propiedades básicas estructurales a ser usados en el diseño estructural de las edificaciones es proporcionada por las cámaras de la construcción, las mismas que utilizan información referencial de materiales de construcción usados en Europa y Norteamérica (INER, 2013). Sin duda, existe gran falta de contenido informativo como artículos o trabajos de investigación sobre la caracterización de materiales de construcción en el país. No existe actualmente una normativa ecuatoriana que especifique los métodos de ensayos a seguir para la determinación de las propiedades térmicas y mecánicas, de los materiales fabricados en la provincia de Pichincha, por lo que se busca aportar con datos al Instituto Nacional de Eficiencia Energética y Energías Renovables (INER), mediante la caracterización con metodologías basadas en proyectos de investigación, libros, artículos, normas INEN, ASTM y procedimientos experimentales. Estos servirán como base para futuros ensayos y prácticas de diseño, para la construcción de edificaciones a nivel nacional. 
Para analizar el intercambio térmico de los materiales de construcción, existen diferentes métodos como el del balance de calor propuesto en la normativa de aire acondicionado, refrigeración y calefacción. Por ejemplo, ASHRAE (American Society of Heating, Refrigerating and Air-conditioning Engineers) que trata de una organización americana que analiza el ahorro energético a través de un método de cálculo para cargas térmicas.

Las propiedades mecánicas de los materiales son importantes de analizar, ya que miden la resistencia a fuerzas o cargas. La respuesta del material ante la deformación tiene relación con la fuerza aplicada, y en caso de ser una resultante excesiva, se producirá la rotura. La resistencia a compresión es la característica principal en materiales de construcción para pared, ya que deben soportar esfuerzos de compresión constantes. Por otro lado, la flexión genera momentos internos cuando se somete a un esfuerzo de flexión, donde se producen tensiones transversales a lo largo del espécimen ensayado, como consecuencia del pandeo. La tracción es el esfuerzo interno que se genera al aplicar dos fuerzas, que actúan en sentidos opuestos hasta la ruptura del material. (Vélez L., 2013). Sobre la resistencia a la compresión, flexión y tracción se puede encontrar el concepto y aplicaciones de estas propiedades con materiales como el concreto y la madera en el libro "Resistencia de materiales" de Pérez (1992).

Trabajos relacionados con esta materia lo realizó Pérez Marín (2005), “Aplicación de nuevos materiales a soluciones de vivienda en Colombia”, determina las propiedades mecánicas a través de ensayos para resistencia tanto a la compresión como a la flexión, descritos en la norma de la Asociación Americana de Ensayos y Materiales ASTM D790; cuya finalidad es aportar con nuevos materiales de construcción para viviendas. En referencia a la caracterización térmica de los materiales, se puede mencionar al artículo internacional "Medición de la Conductividad Térmica de Algunos Materiales Utilizados en Edificaciones" elaborado por Lira C., Gonzales R. y Méndez E. (2008), donde se realizan ensayos de conductividad térmica a bloques de concreto, utilizando el Aparato de placa caliente con guarda (APCG) con flujo de calor permanente. El trabajo de (Moreno, C., Cañizares, O., 2011) donde estudia concreto reforzado con fibras. También otros artículos como que miden resistencia mecánica (Carrera Hidalgo A., 2015), o conductividad térmica (Cuitiño G., Esteves A., Maldonado G., Rotondaro R., 2015).

Este estudio será ejecutado a partir de bloques de concreto obtenidos de diferentes lugares de la provincia de Pichincha del Ecuador, como empresas o viviendas que producen artesanalmente. Se obtendrán las probetas respectivas de los lugares mencionados para los ensayos de resistencia a la compresión contenido de humedad, conductividad térmica, que se efectuarán para obtener las características que permitirán realizar un análisis estadístico y obtener información que servirá para mejorar las futuras edificaciones, brindando comodidad en un ambiente energéticamente eficiente con el ecosistema.

\section{Metodología y materiales}

El bloque de concreto está elaborado con cemento Portland y diferentes áridos gruesos y finos como la grava, arena, arcilla cocida, piedra pómez, piedra partida, granulados volcánicos, escorias y otros materiales inorgánicos. (INEN 638, 2014). Las paredes de los bloques no deben tener un espesor menor a $25 \mathrm{~mm}$ de acuerdo al tipo de bloque A y B; mientras que para los tipos 
de bloques C, D, E, el espesor de paredes no debe ser menor a $20 \mathrm{~mm}$, como se muestra en la tabla 1 .

Tabla 1. Dimensiones de los bloques. Tomado de (INEN 638, 2014).

\begin{tabular}{|c|c|c|c|c|c|c|}
\hline \multirow[t]{2}{*}{ Tipo } & \multicolumn{3}{|c|}{ Dimensiones nominales (cm) } & \multicolumn{3}{|c|}{ Dimensiones efectivas (cm) } \\
\hline & Largo & Ancho & Alto & Largo & Ancho & Alto \\
\hline$A, B$ & 40 & $20,15,10$ & 20 & 39 & $19,14,9$ & 19 \\
\hline C, D & 40 & $10,15,20$ & 20 & 39 & $9,14,19$ & 19 \\
\hline $\mathbf{E}$ & 40 & $10,15,20,25$ & 20 & 39 & $9,14,19,24$ & 20 \\
\hline
\end{tabular}

Los bloques son paralelepípedos que poseen uno o varios huecos transversales en su interior, de manera que del 50 al $75 \%$ sea material sólido. (Moreno, C., Cañizares, O., 2011). Es utilizado generalmente en la construcción de viviendas y edificaciones. El bloque analizado en esta investigación es de $15 \mathrm{~cm}$ de ancho, que se utiliza con más frecuencia en construcciones de uno y dos pisos.

Para este trabajo, se realizaron diferentes ensayos destructivos y no destructivos con probetas de concreto. La obtención del material para los ensayos, fue de diferentes productoras en localidades como Amagüaña, El tingo y Sangolqui. A continuación, en la tabla 1, se muestran las características de los lotes de concreto, obtenidos en diferentes ubicaciones de la provincia de Pichincha.

Tabla 2. Características de los lotes concreto de concreto

\begin{tabular}{lll}
\hline Lote & Ubicación & Composición / Tipo \\
\hline 1 & Sangolqui & $\begin{array}{l}\text { Granulado volcánico, cemento } \\
\text { portland, cal, arena, agua, } \\
\text { aditivos, agregados generales. }\end{array}$ \\
& & \\
& Amagüaña & $\begin{array}{l}\text { Cascajo de Piedra pómez, } \\
\text { cemento portland, cal, arena, } \\
\text { agua, aditivos, agregados } \\
\text { generales. } \\
\end{array}$ \\
& El Tingo & $\begin{array}{l}\text { Piedra partida, cemento } \\
\text { portland, cal, arena, agua, } \\
\text { aditivos, agregados generales. }\end{array}$ \\
& \\
& \\
& \\
& \\
&
\end{tabular}

Para los ensayos mecánicos destructivos se utilizó la maquina electro-hidráulica universal del laboratorio de la Universidad Internacional SEK (UISEK), que se muestra en la figura 2. Esta máquina adopta un cilindro de aceite destinado al control del ajuste descendente que se utiliza principalmente para el metal, la tensión no metálica, la compresión, la flexión y tracción. 
Puede generar una fuerza máxima de $600 \mathrm{kN}$ en los ensayos, para cumplir con los requisitos de normas ASTM, ISO y otros estándares internacionales. La máquina es adecuada para la metalurgia, construcción, industria ligera, aviación, el espacio, materiales, instituciones terciarias, unidades de investigación científica, y otros. Los resultados que se obtuvieron de las gráficas de los ensayos, para los materiales seleccionados, se muestran como una curva de Carga vs Desplazamiento (Fuerza vs Deformación o Esfuerzo vs Deformación).

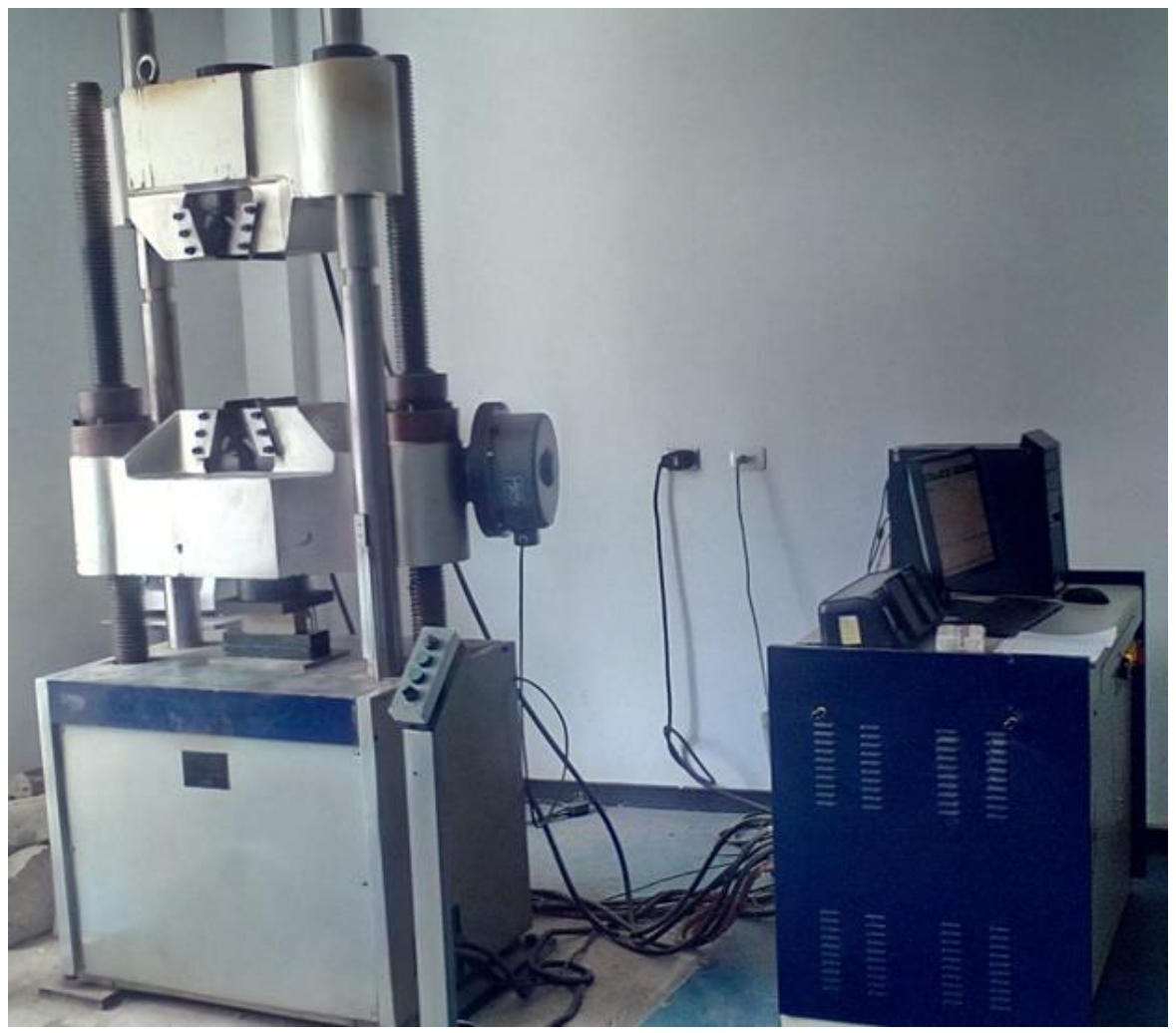

Figura 2. Máquina de ensayos universal de la UISEK.

Se prepararon 6 probetas para cada ensayo, de las cuales 1 se utiliza para medir la carga máxima que se usa de referencia para los ensayos de compresión. Las otras 5 serán las analizadas bajo una norma seleccionada, que será de base para el muestreo con las dimensiones adecuadas para cada prueba. Para el bloque de concreto se ensayaron 5 especímenes a compresión de los tres lotes diferentes, y 4 especímenes de cada lote para ensayos térmicos. Para la determinación de la resistencia a la compresión se siguió el procedimiento estándar NTE INEN 639:2012.

\section{Ensayo de compresión del bloque de concreto}

Se basa en aplicar una carga progresiva de compresión a un bloque, hasta determinar su resistencia máxima admisible (rotura o falla) y registrar su valor. De acuerdo a la normativa se deben ensayar tres especímenes, pero en esta investigación se realizó la prueba de cinco especímenes, para tener más datos. Adicionalmente un espécimen se ensaya para tomar de referencia la carga máxima. Todos los especímenes se deben ensayar con las celdas en posición vertical, excepto para las unidades especiales que se colocan de manera horizontal. Todos los especímenes tienen que estar libres de humedad visible antes de proceder con el ensayo. Se 
aplica la carga a cualquier velocidad aproximadamente hasta la mitad de la carga máxima admisible, para luego aumentar la velocidad gradualmente hasta la falla en un intervalo de 1 a 2 minutos. Este ensayo se inició con uva velocidad aplicada de $0.6 \mathrm{~mm} / \mathrm{min}$ hasta la mitad de la carga máxima y se fue aumentando la velocidad $0.1 \mathrm{~mm} / \mathrm{min}$ progresivamente hasta la falla según la norma. INEN 639, 2012).

Para calcular la resistencia a la compresión se utilizan las ecuaciones siguientes ecuaciones en base al área de la superficie seleccionada, la resistencia a la compresión del área neta (MPa), ecuación (1):

$$
R_{\text {neta }}=\frac{P_{\max }}{A n}
$$

Donde $\mathrm{P} \_$max es la carga máxima de compresión (N) y An es el área neta del espécimen ( 【mm】^2). El área neta se calcula con la ecuación (2):

$$
\mathrm{An}=\mathrm{L} * \mathrm{~W}
$$

Dónde: An es el área neta de la fracción o del espécimen entero ( \mm】^2), L es la longitud promedio de la fracción o del espécimen entero $(\mathrm{mm})$, W es el ancho promedio de la fracción o del espécimen entero $(\mathrm{mm})$.

La resistencia a la compresión del área bruta (Mpa), se calcula mediante (3):

$$
R_{\text {bruta }}=\frac{P_{\max }}{A g}
$$

Donde P_max es la carga máxima de compresión (N), Ag es el área bruta del espécimen ( 【mm】^2). El área bruta se calcula con la ecuación (4):

$$
\mathrm{Ag}=\mathrm{L} * \mathrm{~W}
$$

Donde Ag es el área bruta del espécimen entero ( \mm $\ 2$ ), L es la longitud promedio del espécimen entero $(\mathrm{mm}), \mathrm{W}$ es el ancho promedio del espécimen entero $(\mathrm{mm})$.

\section{Ensayo de contenido de humedad}


Para realizar este ensayo se usan tres especímenes según la norma, pero en este caso se utilizaron cinco. (INEN 639, 2012). Los valores que se calculen para la absorción y contenido de humedad de especímenes cortados, deben ser tomados como representaciones de los especímenes enteros. Para la saturación se sumerge en agua los especímenes a una temperatura entre 16 y $27^{\circ} \mathrm{C}$ durante un lapso de 24 a 28 horas. Se retiran del agua y se dejan escurrir durante un minuto, luego se retira el agua visible con un paño húmedo y se determina la masa Ms (masa del espécimen saturado). Luego se procede con el secado, que consiste en secar todos los especímenes en un horno ventilado entre 100 y $115^{\circ} \mathrm{C}$, durante un tiempo de al menos 24 horas, hasta que la masa del espécimen no disminuya en más del $0.2 \%$ en determinaciones sucesivas a intervalos de 2 horas, respecto a la última determinación. Se registra el valor de los especímenes secos como Md (masa del espécimen seco al horno).

El contenido de humedad se calcula en el momento en el que se realiza el muestreo y se pesa la masa inicial Mr de los especímenes tal como se reciben. Para su cálculo se utiliza la ecuación (5):

$$
C H=\frac{M r-M d}{M s-M d} * 100
$$

Donde Mr es la masa del espécimen tal como se recibe $(\mathrm{kg})$, Md es la masa del espécimen seco al horno $(\mathrm{kg})$, Ms es la masa del espécimen saturado $(\mathrm{kg})$.

\section{Ensayo de conductividad térmica.}

Los ensayos para los materiales en esta investigación se realizaron con la máquina de medición de conductividad térmica de placa caliente, Lambda-Messtechnik $\lambda$-Meter EP500e, Versión C, que se muestra en la figura 3. 


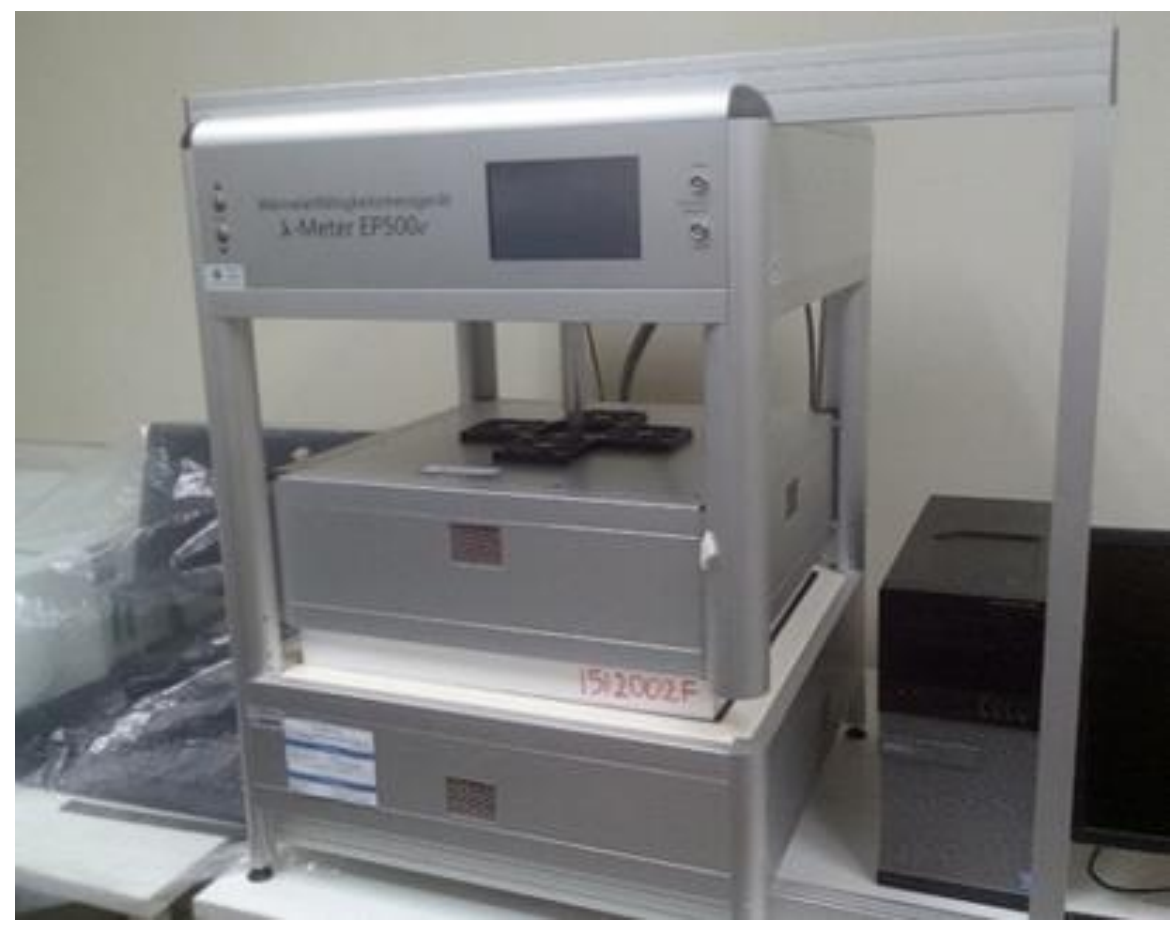

Figura 3. Máquina de ensayos térmicos.

Un APCG funciona basándose en el principio de transferencia de calor por conducción unidireccional y bajo condición de estado estable, entre dos placas frías y una caliente central, en estado permanente para determinar la conductividad térmica con la ecuación (6):

$$
\lambda=\frac{q * L}{\Delta T * A}
$$

Donde $\lambda$ es la conductividad térmica del espécimen $(\mathrm{W} / \mathrm{m} \mathrm{K})$, q es la rapidez del flujo de calor a través del espécimen (W), $\mathrm{L}$ es el espesor de la muestra (m), $\Delta \mathrm{T}$ es la diferencia de temperaturas a través del espécimen $\left({ }^{\circ} \mathrm{C}\right.$ o K $), A$ es el área de la sección transversal $\left(\mathrm{m}^{\wedge} 2\right)$.

El flujo de calor se obtiene de la medición de la corriente y el voltaje de la resistencia eléctrica que provee el calor, ecuación (7):

$$
q=I * V
$$

Donde q es la rapidez del flujo de calor a través del espécimen (W), k es la conductividad térmica $(\mathrm{W} / \mathrm{m} \mathrm{K}), \Delta \mathrm{T}$ es la diferencia de temperaturas a través del espécimen $\left({ }^{\circ} \mathrm{C}\right.$ o $\left.\mathrm{K}\right)$, A es el área de la sección transversal $\left(\mathrm{m}^{\wedge} 2\right)$, L es el espesor de la muestra $(\mathrm{m})$.

Para la obtener el valor de la conductividad térmica, los especímenes deberán estar en estado estable para realizar las mediciones de flujo de calor, espesor y temperaturas fijas de las superficies de ambas caras del espécimen. (ISO 8302, 1991). El rango de conductividad 
permitido según el procedimiento es de 0.002 a $2.5 \mathrm{~W} / \mathrm{m} \mathrm{K}$. Las temperaturas medias para el ensayo son desde $-10^{\circ} \mathrm{C}$ hasta $50^{\circ} \mathrm{C}$, con una diferencia de temperaturas de $15^{\circ} \mathrm{C}$ entre las placas. Las muestras deben ser composiciones homogéneas de forma cuadrada, con dimensiones mínimas de 150 x 150 『mm】^2 y con espesores desde $10 \mathrm{~mm}$ hasta $200 \mathrm{~mm}$.

\section{Resultados}

A continuación, se presentan los resultados de los ensayos de comprensión, contenido de humedad y conductividad.

\section{Resultados de ensayo de compresión}

En la figura 4, se muestra los ensayos realizados a las seis probetas de bloques de concreto. Como se aprecia en la gráfica, la línea con mayor desplazamiento y mayor carga es la probeta de referencia P6C1ref, que fue la sexta probeta ensayada. Dicha probeta fue utilizada para obtener la carga máxima de $102,66 \mathrm{kN}$ que se usa como valor base para el resto de especímenes.

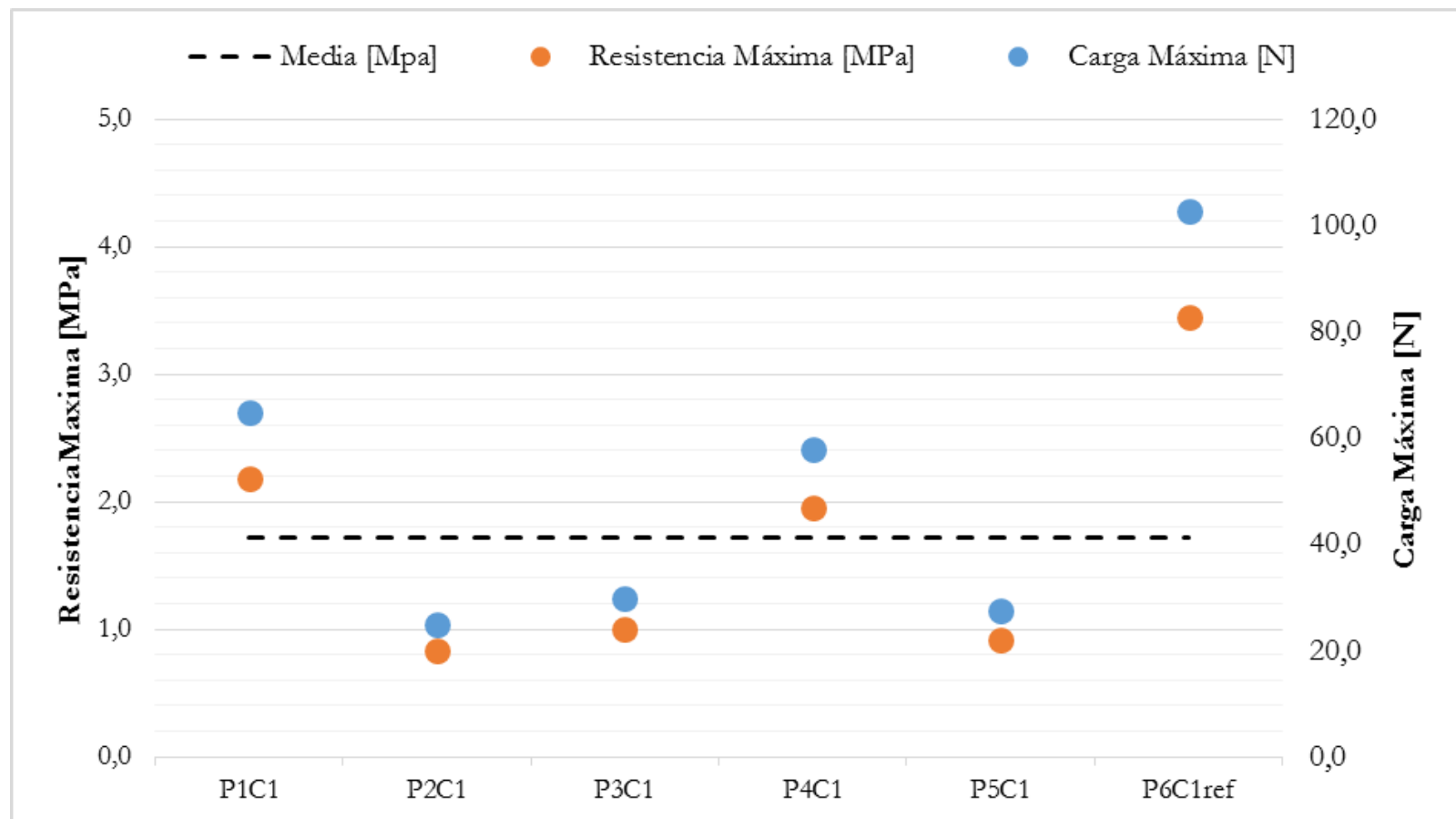

Figura 4. Grafica Carga vs Resistencia resultante del ensayo de compresión del bloque de concreto del sector de Amagüaña.

La tabla 3, muestra el promedio de la resistencia neta a la compresión del bloque de concreto en el sector de Amagüaña, que fue de 1,27 MPa. Según la norma (NTE INEN 643, 2014) la resistencia a compresión en bloques huecos de concreto no soportantes será de 3,5 MPa para unidades individuales y $4 \mathrm{MPa}$ para el promedio de cinco bloques huecos de concreto ensayados.

Tabla 3. Resultados del ensayo de compresión para el bloque de concreto de Amagüaña. 


\section{Compresión del bloque de concreto sector Amagüaña}

\begin{tabular}{cccccc}
\hline \multicolumn{5}{c}{ Compresión del bloque de concreto sector Amagüaña } \\
\hline $\mathbf{N}^{\mathbf{0}}$ & Espécimen & $\begin{array}{c}\text { Área neta, } \mathrm{A}_{\mathrm{n}} \\
\left(\mathrm{mm}^{2}\right)\end{array}$ & Carga máxima, $\mathrm{P}_{\max }(\mathrm{N})$ & $\begin{array}{c}\text { Resistencia, } \\
(\mathrm{MPa})\end{array}$ & $\begin{array}{c}\text { Resistencia, }, \mathrm{R}_{\text {neta }} \\
\left(\mathrm{kg} / \mathrm{cm}^{2}\right)\end{array}$ \\
& & & & \\
$\mathbf{1}$ & P1C1 & 29790 & 64840 & 2,1766 & 22,1949 \\
$\mathbf{2}$ & P2C1 & 29950 & 25010 & 0,8351 & 8,5153 \\
$\mathbf{3}$ & P3C1 & 29930 & 29830 & 0,9967 & 10,1631 \\
$\mathbf{4}$ & P4C1 & 29760 & 57970 & 1,9479 & 19,8633 \\
$\mathbf{5}$ & P5C1 & 29800 & 27440 & 0,9208 & 9,3896 \\
$\mathbf{6}$ & P6C1ref & 29750 & 102660 & 3,4508 & 35,1881 \\
& & Media & 1,27 & 12,91 \\
& & Desviación estándar & 0,635 & 6,473 \\
\hline
\end{tabular}

En la figura 5, se muestran las gráficas de las cinco probetas sometidas a compresión donde se puede observar que el espécimen P2C2 y P4C2 no alcanzan la resistencia deseada en comparación de las otras tres. Esto ocurre cuando el contenido de humedad está presente en algunas zonas del bloque probablemente por el lugar de almacenaje.

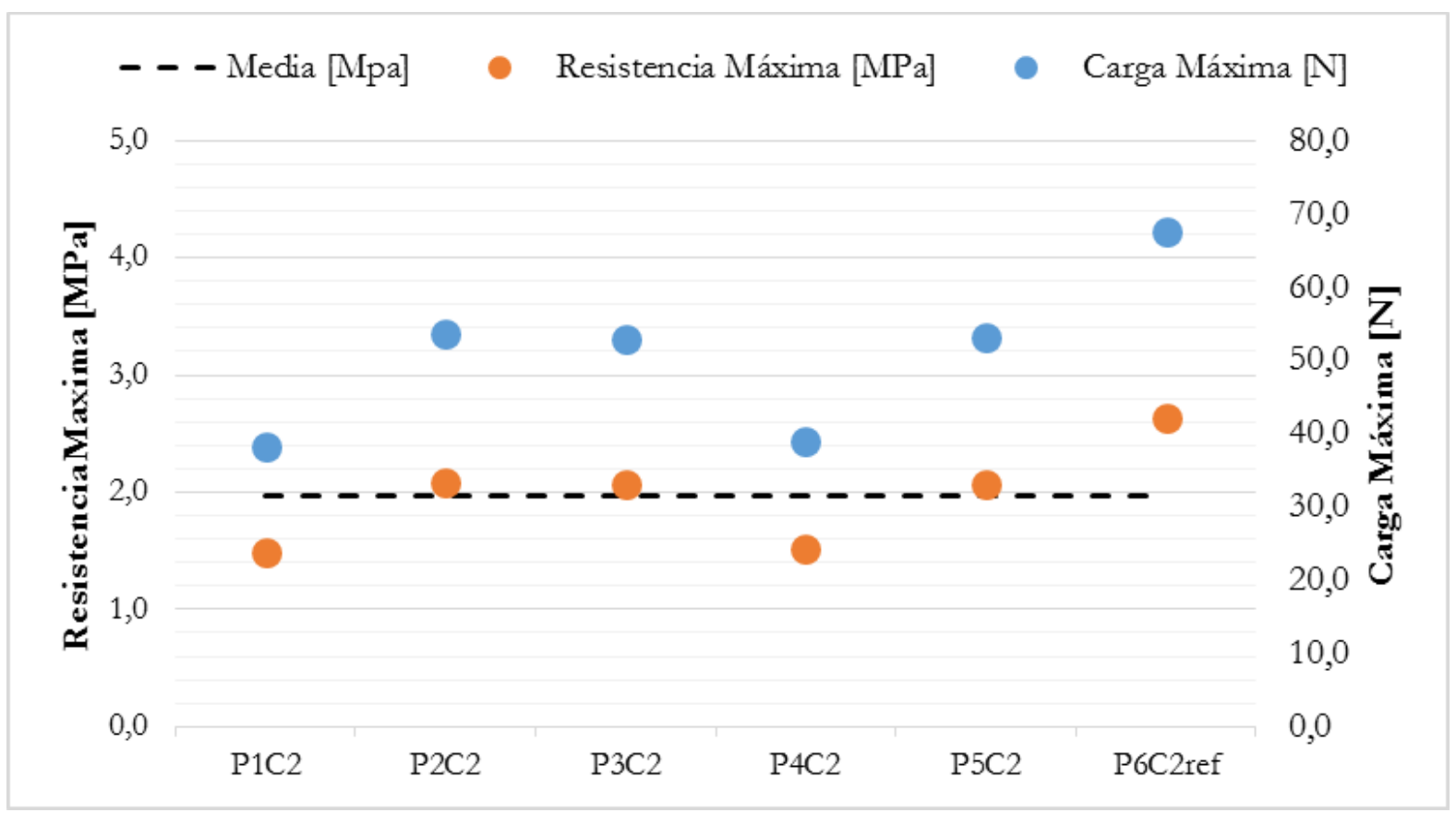

Figura 5. Grafica Carga vs Resistencia resultante del ensayo de compresión del bloque de concreto del sector El Tingo. 
La tabla 4, muestra el promedio de la resistencia neta a la compresión del bloque de concreto en el sector de El Tingo, que fue de $21,65 \mathrm{Kg} / \mathrm{cm} 2$. El valor de la desviación estándar muestra que existe una dispersión máxima de $0,114 \mathrm{Kg} / \mathrm{cm} 2$, respecto a al valor de los especímenes con mayor resistencia.

Tabla 4. Resultados del ensayo de compresión para el bloque de concreto de El Tingo. Compresión del bloque de concreto sector El Tingo

\begin{tabular}{llllll}
\hline \multicolumn{5}{c}{ Compresión del bloque de concreto sector El Tingo } \\
\hline $\begin{array}{l}\mathbf{N} \\
\mathbf{0}\end{array}$ & $\begin{array}{l}\text { Espécime } \\
\mathrm{n}\end{array}$ & $\begin{array}{l}\text { Área neta, } \\
\mathrm{A}_{\mathrm{n}}\left(\mathrm{mm}^{2}\right)\end{array}$ & $\begin{array}{l}\text { Carga máxima } \\
\mathrm{P}_{\max }(\mathrm{N})\end{array}$ & $\begin{array}{l}\text { Resistencia }, \\
\mathrm{R}_{\text {neta }}(\mathrm{MPa})\end{array}$ & $\begin{array}{l}\text { Resistencia } \\
\mathrm{R}_{\text {neta }}\left(\mathrm{kg} / \mathrm{cm}^{2}\right)\end{array}$ \\
$\mathbf{1}$ & $\mathrm{P} 1 \mathrm{C} 2$ & 25650 & 38110 & 1,8858 & 15,1507 \\
$\mathbf{2}$ & $\mathrm{P} 2 \mathrm{C} 2$ & 25650 & 53560 & 2,0881 & 21,2929 \\
$\mathbf{3}$ & $\mathrm{P} 3 \mathrm{C} 2$ & 25650 & 52870 & 2,0612 & 21,0186 \\
$\mathbf{4}$ & $\mathrm{P} 4 \mathrm{C} 2$ & 25650 & 38940 & 1,8181 & 15,4807 \\
$\mathbf{5}$ & $\mathrm{P} 5 \mathrm{C} 2$ & 25650 & 53140 & 2,0717 & 21,1259 \\
$\mathbf{6}$ & P6C2ref & 25650 & 67534 & 2,6329 & 26,8483 \\
\multicolumn{7}{l}{ Media } & & & 2,12 & 21,65 \\
\multicolumn{7}{l}{ Desviación estándar } & & 0,114 & 1,197 \\
\hline
\end{tabular}

En la figura 6, se puede observar que los especímenes $\mathrm{P} 2 \mathrm{C} 3$ y $\mathrm{P} 4 \mathrm{C} 3$ tienen menor resistencia, debido a la humedad que pueden presentar los bloques.

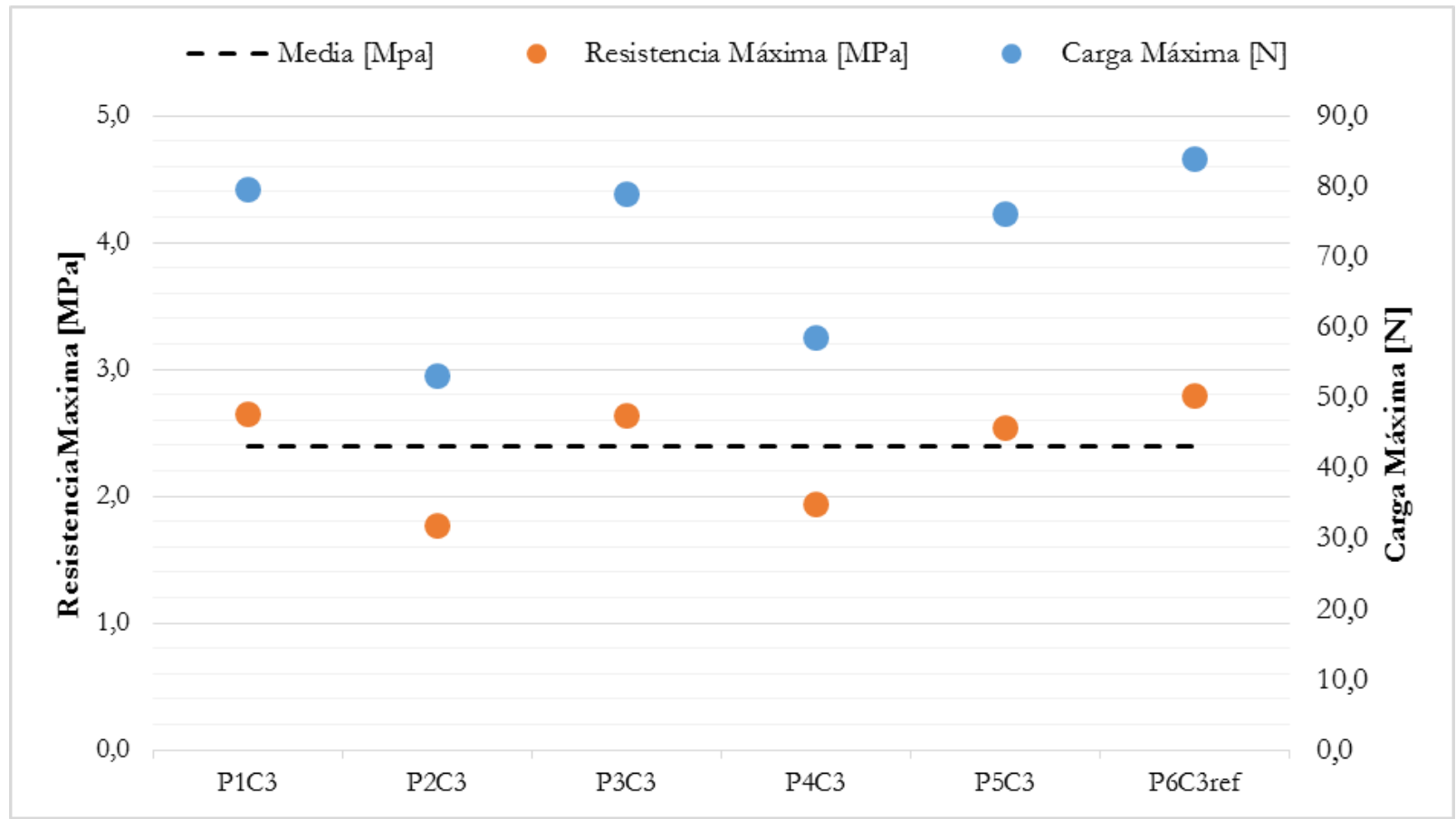


Figura 6. Grafica Carga vs Resistencia resultante del ensayo de compresión del bloque de concreto del sector Sangolqui.

En la tabla 6, se muestra el promedio de la resistencia neta a la compresión del bloque de concreto en el sector de Sangolqui, que fue de 24,21 Kg/cm2. El trabajo de (Hidalgo A., 2015) reporta un valor de $24,19 \mathrm{Kg} / \mathrm{cm} 2$ para la resistencia a compresión en bloques huecos de hormigón, y considera que es un valor apropiado ya que supera los $2 \mathrm{MPa}$.

Tabla 6. Resultados del ensayo de compresión para el bloque de concreto de Sangolqui.

\begin{tabular}{llllll}
\hline \multicolumn{6}{l}{ Compresión del bloque de concreto sector Sangolqui } \\
\hline $\mathbf{N}^{\mathbf{0}}$ & Espécimen & $\begin{array}{l}\text { Área neta, } \mathrm{A}_{\mathrm{n}} \\
\left(\mathrm{mm}^{2}\right)\end{array}$ & $\begin{array}{l}\text { Carga máxima }, \mathrm{p} \mathrm{M}_{\mathrm{ax}} \\
(\mathrm{N})\end{array}$ & $\begin{array}{l}\text { Resistencia, } \\
(\mathrm{Mpa})\end{array}$ & $\begin{array}{l}\text { Resistencia, } \mathrm{R}_{\text {neta }} \\
\left(\mathrm{kg} / \mathrm{cm}^{2}\right)\end{array}$ \\
$\mathbf{1}$ & P1C3 & 30000 & 79580 & 2,6527 & 27,0498 \\
$\mathbf{2}$ & P2C3 & 30000 & 53140 & 1,7713 & 18,0626 \\
$\mathbf{3}$ & P3C3 & 30000 & 79030 & 2,6343 & 26,8628 \\
$\mathbf{4}$ & P4C3 & 30100 & 58540 & 1,9449 & 19,8320 \\
$\mathbf{5}$ & P5C3 & 30000 & 76180 & 2,5393 & 25,8941 \\
$\mathbf{6}$ & P6C3ref & 30080 & 84040 & 2,7939 & 28,4898 \\
\multicolumn{2}{l}{ Media } & & & 2,37 & 24,21 \\
\multicolumn{7}{l}{ Desviación estándar } & & 0,418 & 4,262 \\
\hline
\end{tabular}

La figura 7, muestra la diferencia de los resultados obtenidos, de la resistencia a la compresión para el bloque de concreto de los diferentes sectores. Se puede observar que los bloques del sector Amagüaña, tienen una diferencia notable en los valores. 


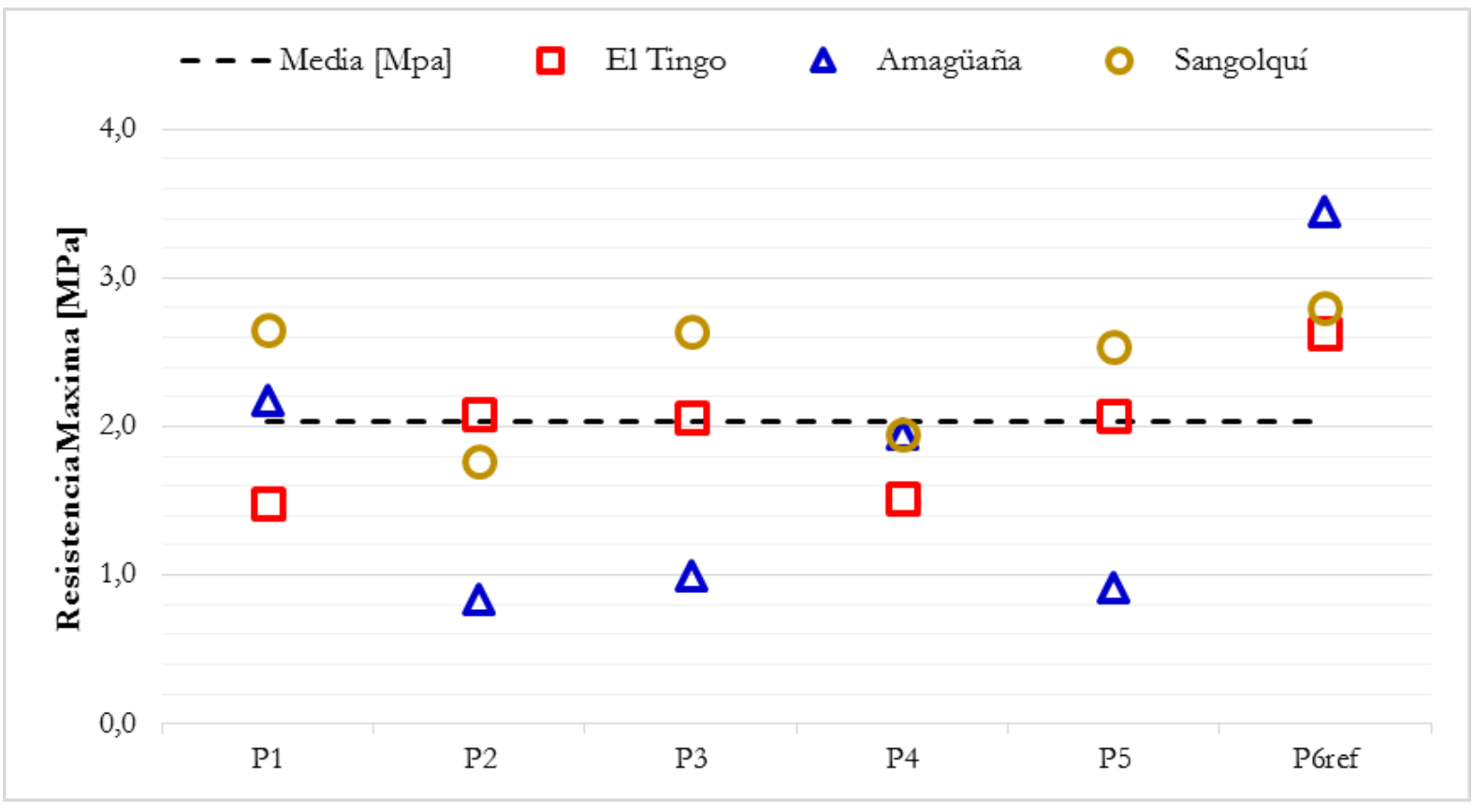

Figura 7. Grafica comparativa de resistencia a compresión en bloques de concreto de diferentes sectores.

\section{Resultados de los ensayos de contenido de humedad}

En la figura 8, se observa el contenido de humedad de cada probeta de bloque de concreto para los sectores de Sangolqui, El Tingo y Amagüaña. Las probetas del último sector mencionado muestran mayor $\mathrm{CH}$ debido a los agregados que poseen.

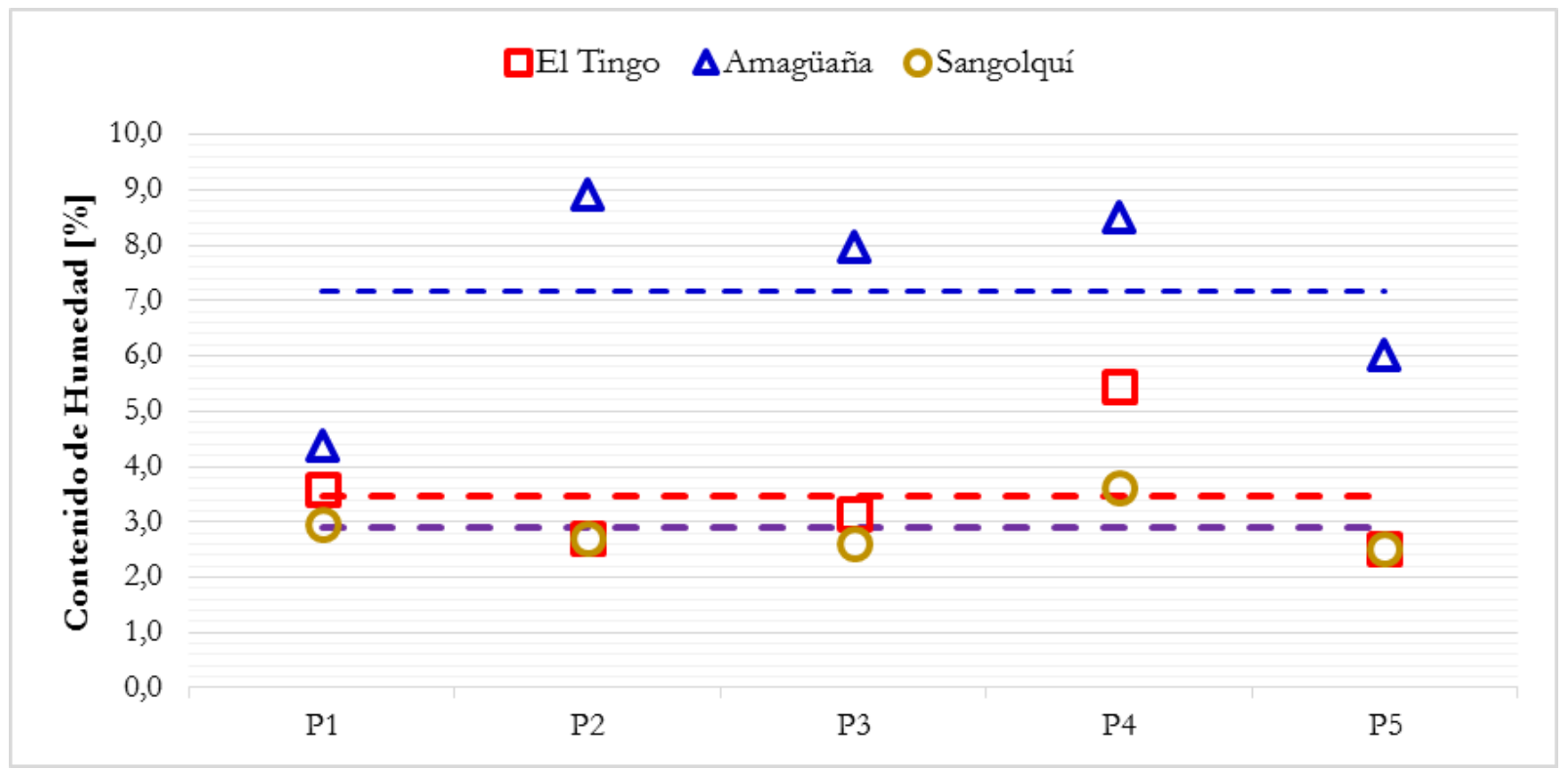

Figura 8. Graficas del contenido de humedad en bloques de concreto de diferentes sectores. 
La tabla 7 muestra el promedio del contenido de humedad de bloques de concreto del sector de Amagüaña que es de 6.93\%. De acuerdo con la norma (NTE INEN 639:2012) el valor no podrá superar el $15 \%$ para que cumpla con los requisitos.

Tabla 7. Resultados del ensayo de contenido de humedad para el bloque de concreto (Amagüaña).

\begin{tabular}{llllll}
\hline & & & & & \\
$\mathrm{N}^{\circ}$ & $\begin{array}{l}\text { Espécime } \\
\mathrm{n}\end{array}$ & $\begin{array}{l}\text { Peso, Mr } \\
(\mathrm{g})\end{array}$ & $\begin{array}{l}\text { Peso, Md } \\
(\mathrm{g})\end{array}$ & $\begin{array}{l}\text { Peso, Ms } \\
(\mathrm{g})\end{array}$ & $\mathrm{CH} \%$ \\
\hline 1 & P1BH1 & 4930 & 4902 & 5540 & 4,38871 \\
2 & P2BH1 & 4950 & 4898 & 5480 & 8,93471 \\
3 & P3BH1 & 4889 & 4835 & 5512 & 7,97637 \\
4 & P4BH1 & 5012 & 4964 & 5528 & 8,51064 \\
5 & P5BH1 & 4895 & 4855 & 5520 & 6,01504 \\
& & & & 6,9324 \\
& & & & & 1,9125 \\
\hline
\end{tabular}

La tabla 8 muestra el promedio del contenido de humedad de bloques de concreto del sector de El Tingo que es de 3,34\%. Se puede notar que la desviación estándar muestra una diferencia representativa respecto a la media. Esto se debe a que un espécimen tuvo más absorción debido a la compactación del material por una mezcla no homogénea.

Tabla 8. Resultados del ensayo de contenido de humedad para el bloque de concreto (EI Tingo).

\begin{tabular}{llllll}
\hline $\mathrm{N}^{\circ}$ & $\begin{array}{l}\text { Espécime } \\
\mathrm{n}\end{array}$ & $\begin{array}{l}\text { Peso, } \mathrm{M}_{1} \\
(\mathrm{~g})\end{array}$ & Peso, $\mathrm{M}_{2}(\mathrm{~g})$ & $\begin{array}{l}\text { Peso, } \mathrm{M}_{3} \\
(\mathrm{~g})\end{array}$ & $\mathrm{CH} \%$ \\
\hline 1 & P1BH2 & 3846 & 3812 & 4761 & 3,58272 \\
2 & $\mathrm{P} 2 \mathrm{BH} 2$ & 3901 & 3876 & 4798 & 2,71150 \\
3 & P3BH2 & 3880 & 3851 & 4775 & 3,13853 \\
4 & P4BH2 & 3887 & 3835 & 4788 & 5,45645 \\
5 & P5BH2 & 3896 & 3873 & 4791 & 2,50545 \\
& & & & 3,3419 \\
& Media & & & 1,1805 \\
\hline
\end{tabular}

La tabla 9 muestra el valor promedio del contenido de humedad de bloques de concreto del sector de Sangolqui que es de 3,13\%. Normalmente existe mayor porcentaje de humedad 
cuando la composición del bloque carece de fibras, ya que las mismas contribuyen en el compactado para evitar la humedad.

Tabla 9. Resultados del ensayo de contenido de humedad para el bloque de concreto (Sangolqui).

\begin{tabular}{llllll}
\hline $\mathrm{N}^{\circ}$ & Espécimen & $\begin{array}{l}\text { Peso, M1 } \\
(\mathrm{g})\end{array}$ & Peso, M2 (g) & Peso, M3 (g) & $\mathrm{CH} \%$ \\
\hline 1 & P1BH3 & 4621 & 4593 & 5542 & 2,95047 \\
2 & P2BH3 & 4612 & 4584 & 5534 & 2,94737 \\
3 & P3BH3 & 4627 & 4602 & 5553 & 2,62881 \\
4 & P4BH3 & 4633 & 4598 & 5564 & 3,62319 \\
5 & P5BH3 & 4648 & 4613 & 5570 & 3,65726 \\
& & & & 3,1352 \\
\multicolumn{7}{r}{ Media } & & & & 0,4564 \\
\hline
\end{tabular}

\section{Resultados de conductividad térmica.}

La figura 9, muestra la conductividad térmica de cada probeta para los tres lotes de bloque de concreto. Como se puede ver en la gráfica, la diferencia de conductividad térmica de los especímenes entre sectores es baja.

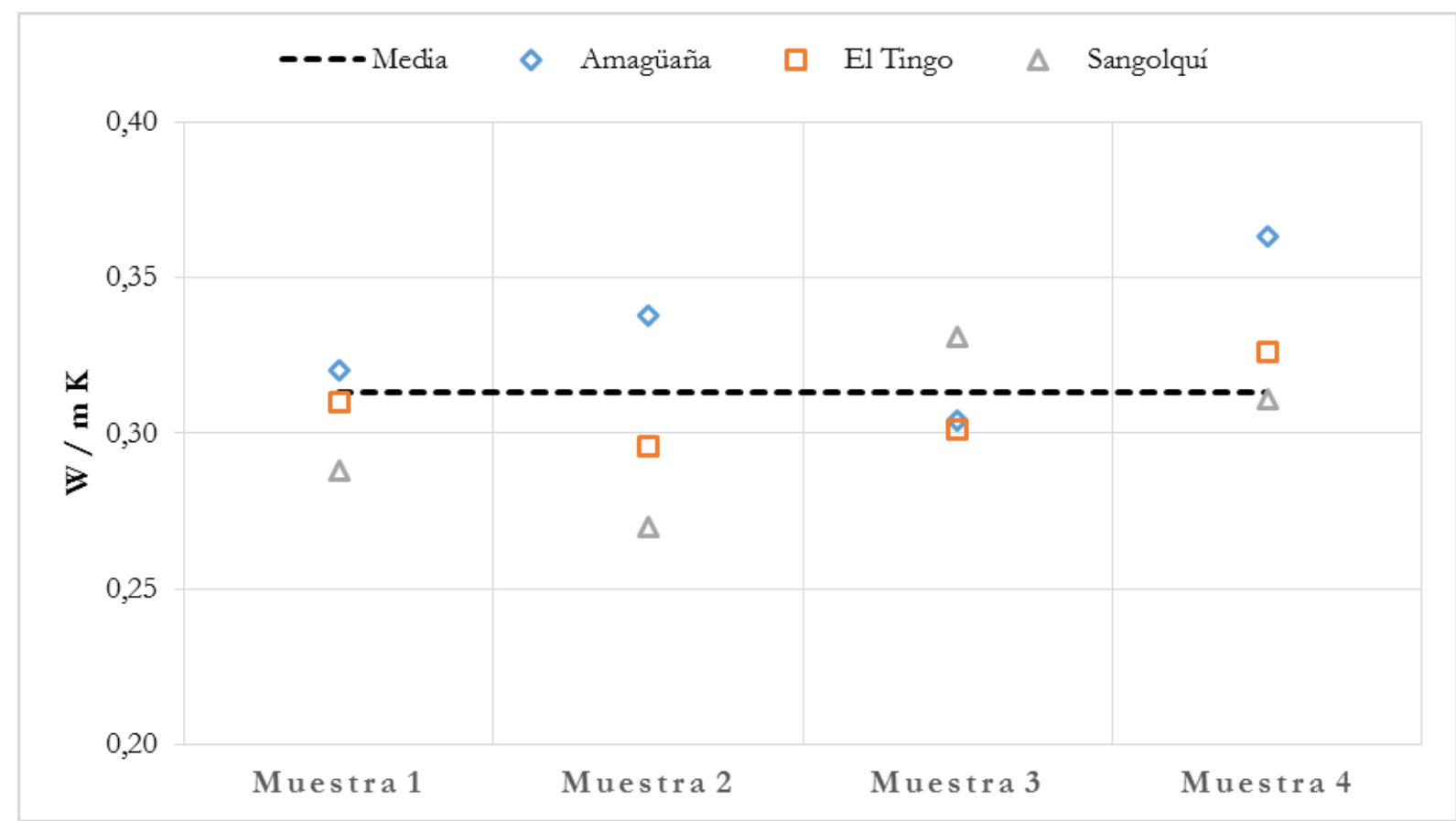

Figura 9. Gráfica comparativa de la conductividad térmica en bloques de concreto de varios sectores.

En la tabla 10, se muestran los resultados promedios de la conductividad térmica y de la resistencia térmica de bloques de hormigón, con 0,312 W/m K y 0,081 m2 K/W respectivamente. 
Los valores promedios de cada lote muestran que los bloques procedentes de Sangolqui son menores, mientras que los bloques procedentes de Amagüaña son mayores.

\section{Tabla 10. Resultados del ensayo de conductividad térmica para los bloques de concreto de las diferentes zonas de Pichincha.}

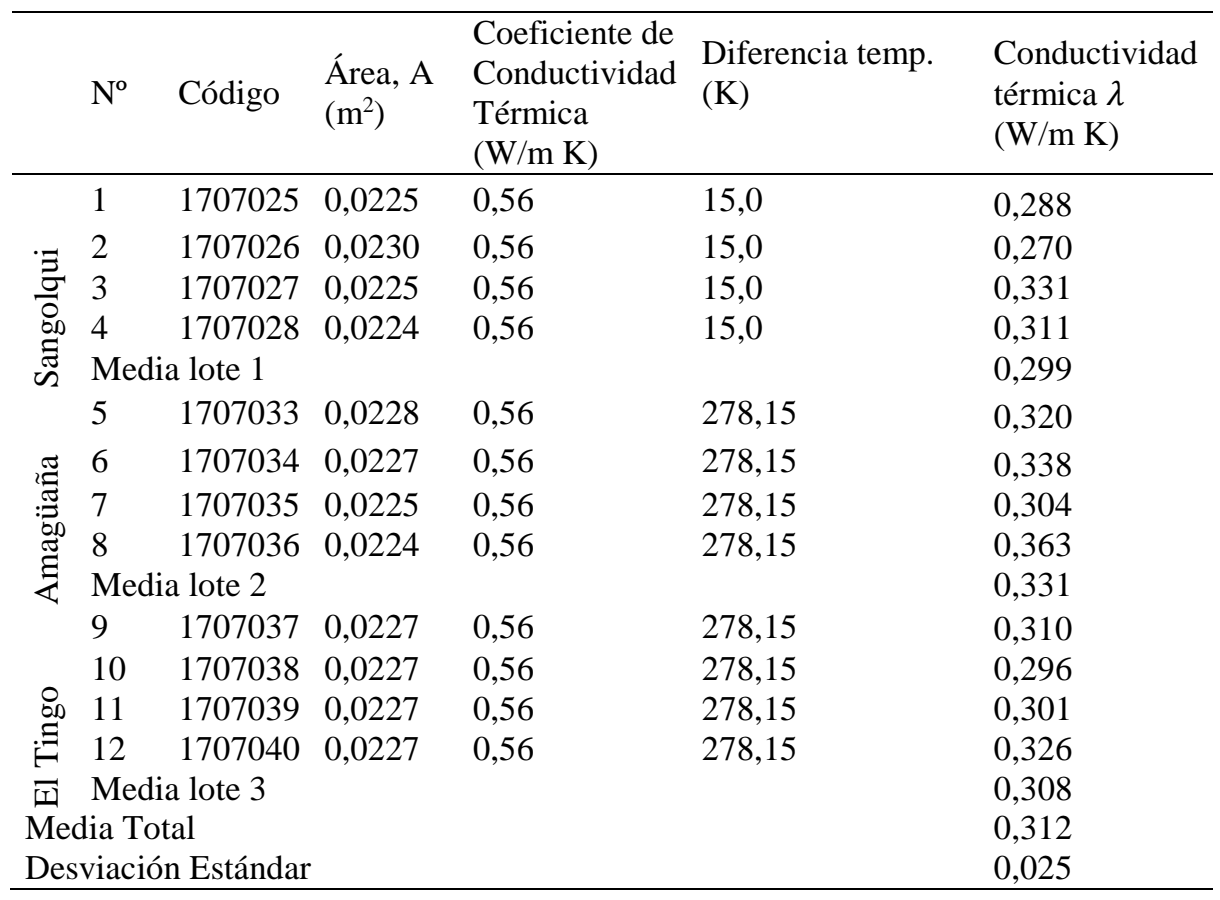

\section{Discusión}

El presente trabajo pretende ayudar en la literatura circundante sobre las características térmicas y mecánicas de los bloques de concreto que se producen en la provincia de Pichincha. De los resultados que se obtuvieron en los ensayos compresión, en los distintos sectores no cumple la muestra de bloques del sector de Amagüaña, debido a la baja resistencia a compresión según la norma (NTE INEN 643, 2014). Como se puede apreciar en el trabajo de (Moreno, C., Cañizares, O., 2011) los resultados de la resistencia a compresión varían de acuerdo a los porcentajes del compuesto de fibra que es de plástico es este caso. Además, se consideran valores aceptables cuando la resistencia a compresión supera el valor de $2 \mathrm{MPa}$, (Hidalgo A., 2015), por lo tanto, los bloques de los sectores de El tingo y Sangolqui si cumplen con los establecido. Los bloques del sector del Tingo tienen una longitud menor a los bloques de los otros dos sectores, generando pérdidas de resistencia a la compresión.

Los resultados obtenidos del contenido de humedad están dentro de los parámetros establecidos. Los datos recopilados por (Moreno, C., Cañizares, O., 2011) muestran el porcentaje del contenido de humedad de bloques de concreto en especímenes con plástico y sin plástico, siendo aceptables en base a lo establecido en la norma (INEN 642). Existe una diferencia insignificante entre los resultados de los sectores de El Tingo y Sangolqui; mientras que el valor promedio de los bloques del sector de Amagüaña es dos veces mayor. Esto se genera cuando los 
bloques aun no alcanzan su punto óptimo de fraguado que es de 28 días y los venden cuando tienen apenas 4 o 5 días.

Los resultados de conductividad térmica para los bloques de hormigón fueron de 0,299 $\mathrm{W} / \mathrm{m} \mathrm{K}$ del lote de muestras de Sangolqui con densidad promedio de 1676,48 Kg/m3, 0,308 W/m $\mathrm{K}$ del lote de muestras de El tingo con densidad promedio de $1633,8 \mathrm{Kg} / \mathrm{m} 3$ y $0,331 \mathrm{~W} / \mathrm{m} \mathrm{K}$ del lote de muestras en Amagüaña con densidad promedio de $1688,3 \mathrm{Kg} / \mathrm{m} 3$. Algunos autores establecen un valor de 1,63 W/m K para muestras con densidad de $2400 \mathrm{Kg} / \mathrm{m} 3$, en el trabajo de (Cuitiño G., Esteves A., Maldonado G., Rotondaro R., 2015). En las normas (ASHRAE 90.1, 2007) también se puede encontrar un valor de $0,88 \mathrm{~W} / \mathrm{m} \cdot \mathrm{K}$ para bloques de concreto con densidad de $1680 \mathrm{Kg} / \mathrm{m} 3$. En base a estos datos los bloques analizados poseen mejores características de conductividad térmica, que los analizados en los trabajos antes mencionados. Por esta razón, el valor promedio de $0,312 \mathrm{~W} / \mathrm{m} \mathrm{K}$ muestra que son materiales con mejor aislamiento térmico

\section{Conclusiones}

Después de analizar los resultados y compararlos con las referencias consultadas en esta investigación, se puede notar la falta de información referente a los materiales de construcción, donde especifiquen valores admisibles especialmente para la conductividad térmica.

Los resultados muestran que los bloques del sector de Amagüaña poseen menor resistencia a compresión en base a la norma INEN. Una causa probable de estos resultados es el dimensionamiento con medidas inferiores a las que se producen generalmente. En cuanto a los bloques de Sangolqui y El Tingo muestran propiedades con valores admisibles.

En los resultados de la conductividad térmica se mostraron valores menores a los de las referencias consultadas. Esto demuestra que tienen mejores características aislantes y pueden ser utilizados para la construcción de muros y paredes.

Después de haber obtenido los resultados en los diferentes ensayos, se puede decir que los valores varían dependiendo del método de elaboración. Donde se utilizan moldes elaborados por las mismas productoras y no constan de una normativa que regule el dimensionamiento de las mimas.

Es recomendable que a partir de estos datos se pueda generar una normativa donde se establezca una metodología concreta para caracterizar materiales constructivos y donde se muestre los valores admisibles para los diferentes ensayos.

Se recomienda a las autoridades correspondientes, crear un ente que regule a las productoras de bloques de concreto, ya que los moldes que se usan producen variaciones en el dimensionamiento de los bloques. 


\section{Bibliografía}

Ansi/Ashrae/Iesna Standard 90.1 (2007). Energy Standard for Buildings except Low-Rise Residential Buildings, SI Edition.

ASTM D143 (2016). Standard test methods for Small Clear Specimens of Timber. Recuperado de http://file.yizimg.com/424653/2013110715144243.pdf

Carrera Hidalgo A. (Junio de 2015). Análisis y desarrollo para la fabricación de bloques de hormigón como aislantes térmicos basados en la biomasa de la cascarilla de cacao apto para construcción en edificaciones. Universidad de las fuerzas armadas.

Commissie, E. A Roadmap for moving to a competitive low carbón economy in 2050. 2011.

Cuitiño, G., Esteves, A., Maldonado, G., Rotondaro, R. (Abril de 2015). Análisis de la transmitancia térmica y resistencia al impacto de los muros de quincha. Informes de la Construcción. Obtenido de http://dx.doi.org/10.3989/ic.12.082.

ECOFYS. Assessment of cost optimal calculations in the context of the EPBD. 2014.

ISO 8302 (Agosto de 1991). Thermal insulation. Determination of steady-state thermal resistance and related properties - Guarded hot plate apparatus.

L. Lira Cortés, González Rodríguez, O. J., Méndez Lango, E. (22 de Octubre de 2008). Medición de la Conductividad Térmica de Algunos Materiales Utilizados en Edificaciones. México: Centro Nacional de Metrología. Recuperado de https://www.cenam.mx/simposio2008/sm_2008/memorias/S4/SM2008-S4C2-1100.pdf

Moreno Cárdenas R., Cañizares Ortega F. (Julio de 2011). Agregado alternativo para fabricación de bloques y adoquines en base a polietileno tereftalato. Escuela Politécnica Nacional.

Norma Básica de la Edificación NBE-CT-79 (Julio de 1979), sobre Condiciones térmica en los edificios. Obtenido de https://w3.ual.es/Depar/proyectosingenieria/descargas/Normas_Edificacion/NBE-CT79.pdf

NORMA E.080 (2013). Diseño y Construcción con tierra reforzada.

Normas técnicas complementarias para diseño y construcción de estructuras de madera (Agosto de 2008). Obtenido de http://cgservicios.df.gob.mx/prontuario/vigente/744.pdf

NTE INEN 638 (2014). Bloques huecos de hormigón. Definiciones, clasificación y condiciones generales.

NTE INEN 639 (2012). Bloques huecos de hormigón. Muestreo y ensayos.

NTE INEN 643 (2014). Bloques huecos de hormigón. Requisitos. 
Pérez, White T. (1992). Resistencia de materiales. Universidad. Ediciones Universidad de Salamanca.

Vélez, Moreno L. (Abril de 2013). Propiedades Materiales de Construcción. Obtenido de http://propiedadesmaterialesdeconstruccin.blogspot.com/2013/04/propiedades-mecanicasde-los-materiales.html 